\title{
CLINICAL FINDINGS AND MANAGEMENT OF PELLUCID MARGINAL DEGENERATION USING SCLERAL LENSES OVER INTACS AND CORNEAL COLLAGEN CROSS-LINKING WITH RIBOFLAVIN
}

By Brad T. Cunningham, OD, FSLS

\begin{abstract}
About the Author
Dr. Brad Cunningham graduated from New England College of Optometry in 2007 and has been serving the United States Army Medical Service Corps ever since. He completed his primary care and contact lens residency from the University of Incarnate Word, Rosenberg School of Optometry and gained his fellowship in scleral lenses (FSLS) in 2017.
\end{abstract}

Correspondence may be directed to Brad Cunningham: bcunning45@gmail.com

Submitted: September 27, 2017. Accepted: December 13, 2017. Published: January 19, 2018.

\begin{abstract}
This case study provides an analysis of an individual affected by pellucid marginal degeneration (PMD). This particular case demonstrates the effectiveness of new scleral lenses over a severe form of PMD that underwent corneal crosslinking (CXL) with riboflavin over intra-stromal corneal ring segments (INTACS). PMD is a rare, progressive, degenerative corneal disease typically characterized as peripheral, bilateral, and inferior ectasia producing a crescent shape. It has been found more commonly in males and appears between the second and fifth decade of life showing no signs of ethnic predilection. Although there is no evidence of the prevalence and etiology of PMD, it may be postulated that PMD is poorly differentiated because this disease is often confused or used interchangeably with keratoconus (KC). KC is a developmental anomaly in which a part of the cornea becomes thinner and bulges forward in a cone-shaped fashion as a result of non-inflammatory stromal thinning. $\mathrm{KC}$ is predominantly bilateral (90\%) and according to recent studies demonstrates a prevalence of $2.3 \%$. The Collaborative Longitudinal Evaluation of Keratoconus (CLEK) study found the genetic predisposition of $\mathrm{KC}$ to be at $13.5 \%$, whereas other studies found it to be between $6-10 \%$. This means most of those found having $\mathrm{KC}$ have no known association with the disease. More importantly, the cause of $\mathrm{KC}$ is still unknown. Regardless, the Federal Drug Administration (FDA) approval of KC treatment using CXL in April 2016 has shown excellent promise of improving the visual potential for patients with this progressive disease. This case demonstrates the usefulness of utilizing scleral gas permeable (GP) contacts over INTACS post CXL to provide optimal, stable vision, comfort and therapy. In addition, this paper shows an innovative way to utilize corneal topography to ensure proper fitting scleral lenses.
\end{abstract}

\section{BACKGROUND}

Patient \#2, a 36-year-old Hispanic male, presented in the optometry clinic on October 14th, 2016 for a medical contact lens evaluation due to presumed advanced keratoconus (KC) in both eyes. This patient was medically retired in 2009 due to his diagnosis of advancing $\mathrm{KC}$ and worsening vision. The patient's last eye exam was a medical contact lens evaluation for his prosthetic replacement of the ocular surface ecosystem (PROSE) lenses by BostonSight ${ }^{\circledR}$, fit by another provider. The patient's presenting scleral GP lenses measured as: OD: BC: 7.9, Dia: $19 \mathrm{~mm}$, Pwr: -9.00D, Seg Height: 5200 um, and OS: BC: 7.9, Dia: 19 mm, Pwr: -7.75D, Seg Height: 5100 um. His

J Cont Lens Res Sci Vol 2(1):1-14; January 19, 2018. @ 2018 Journal of Contact Lens Science and Research. All rights reserved. This article is distributed under the terms of the Creative Commons Attribution-Non Commercial 4.0 International License. 
vision was recorded as 20/25 in the right eye with no improvement with an over-refraction and 20/20 in the left eye.

During a routine contact lens follow-up, the patient was properly educated by his team of eye doctors about other procedures including the option of INTACS and CXL. Although he appeared to be pleased with the results of the PROSE lenses, he became intolerant of the scleral contacts from discomfort and vision. U1timately, he wanted to avoid penetrating keratoplasty procedures. The patient made every effort to prevent any corneal progression. He received 2 INTACS OU on June $9^{\text {th }}, 2016$ and CXL with riboflavin OU on August $9^{\text {th }}, 2016$. This elective, off-label procedure was conducted by a private practice corneal ophthalmologist.

The patient's pre-operative vision was best corrected to OD 20/25 and OS 20/20 with contacts. The patient's corneal topography (Figure 1A and 1B) by the Oculus,
Pentacam ${ }^{\circledR}$ demonstrated high irregular astigmatism caused by advanced PMD. Severe steepening $>60 \mathrm{D}$ and apical thinning was observed in both eyes. The pre-operative comprehensive dilated exam found no significant posterior segment findings.

The patient's post-operative best corrected acuity was OD 20/20 - and OS 20/20 with contacts. His last eye exam was approximately 6 months prior with his corneal specialist and no additional significant finding was reported. His medical and family history was unremarkable. No other family member was known to suffer from $\mathrm{KC}$ or any corneal ectasia. The patient reported good vision with the PROSE lenses however he complained "seeing the fluid" and when light emerges from the sides he becomes distracted. Since the patient became intolerant of the lenses, he was offered to be fit with a different scleral lens design. He accepted and was scheduled for a new fitting.

FIG. 1A Pre-operative OD topography.
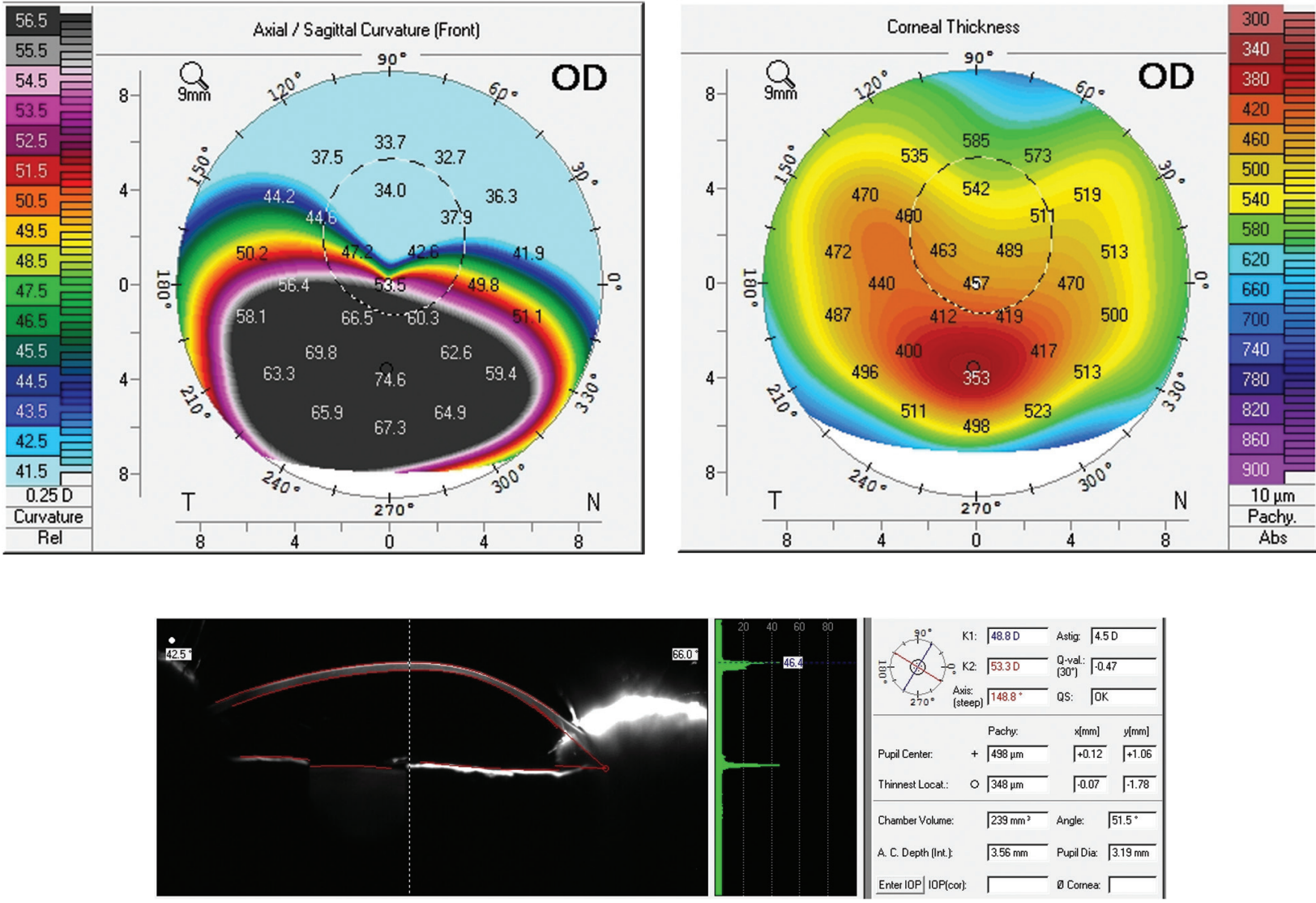

J Cont Lens Res Sci Vol 2(1):1-14; January 19, 2018. @ 2018 Journal of Contact Lens Science and Research. All rights reserved. This article is distributed under the terms of the Creative Commons Attribution-Non Commercial 4.0 International License. 
FIG. 1B Pre-operative OS topography.
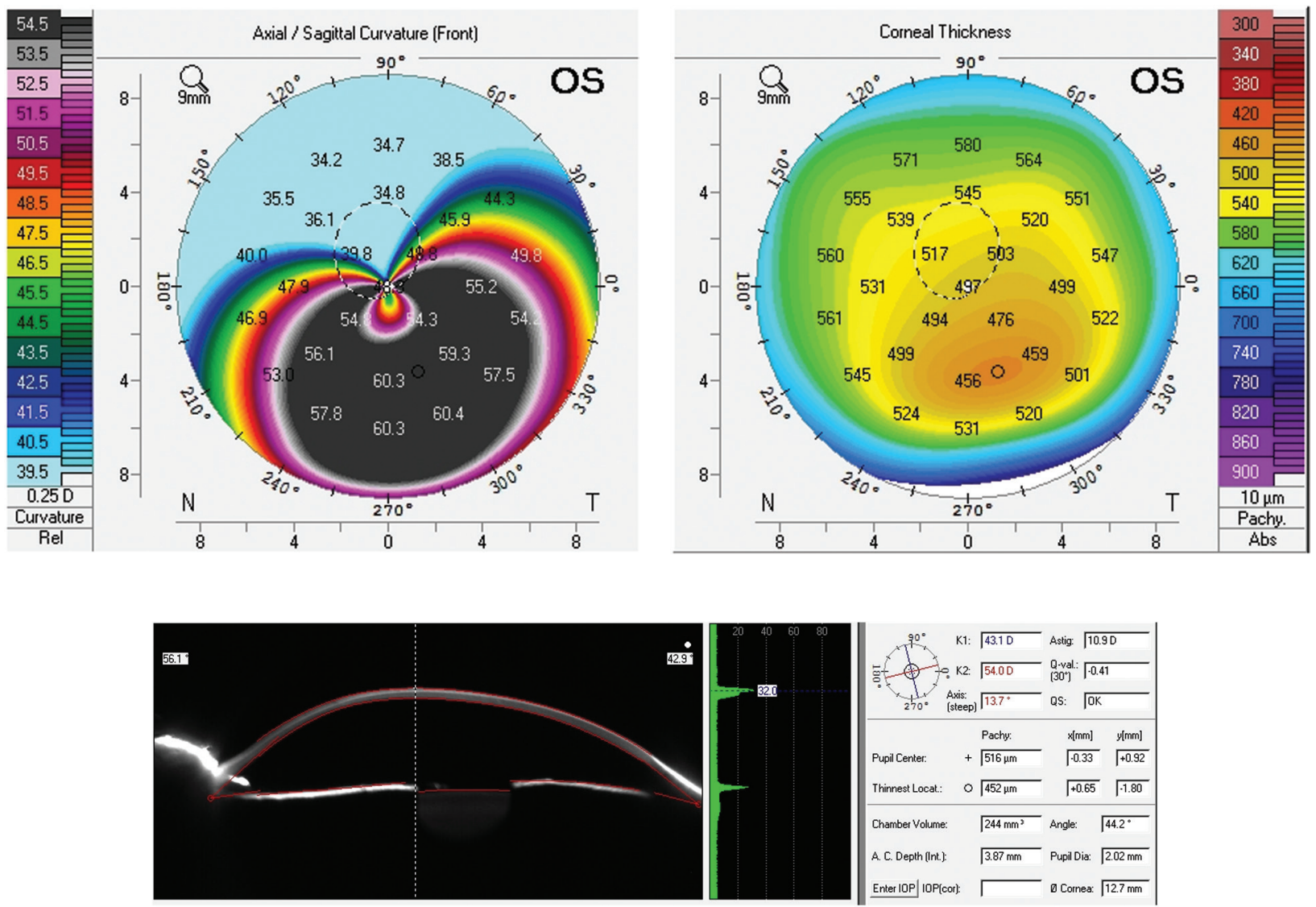

\section{INITIAL EXAM AND GP FITTING}

On October $14^{\text {th }}, 2016$, the patient was fitted with the Zenlens by Alden Optical. Uncorrected visual acuity was OD 20/100, pin-holed to $20 / 30$ and OS $20 / 400$ pin-holed to 20/100. Uncorrected near vision was OD 20/30 and OS 20/50. Manifest refraction in the right eye was $-5.25-5.50 \times 030$ to $20 / 30-2$ and $-5.75-4.50 \times 056$ to $20 / 20-$ in the left eye. The patient reported uncomfortable distortion of all fine detail and ghosting of the letters in both eyes with best spectacle correction. His pupils were equally round and reactive to light with no afferent pupil defect observed OU. Confrontation fields were full to finger count OU. His extraocular muscles were unrestricted in all gazes and cover test demonstrated orthophoria at distance and 5 prism diopters of exophoria at near. His intraocular pressure was $10 \mathrm{mmHg}$ OD and $8 \mathrm{mmHg}$ OS using non-contact tonometry at 1:14p.m.

Slit lamp examination revealed $1+$ conjunctival injection of both eyes, significant inferior stromal thinning with striae and small mild round scarring slightly inferior of both corneas. The 4 INTACS appeared well placed at one and seven o'clock OD and five and eleven o'clock OS. No signs of complication observed. About $1 \mathrm{~mm}$ of temporal limbal neovascularization of encroachment in the left eye only was observed. Both eyes exhibited scattered 1+ punctate epithelial keratopathy with fluorescein. Lids and lashes were clear; anterior chambers were deep and quiet. Irises were flat and brown and lenses were clear in both eyes. All other ocular structures were normal.

Corneal topography (Figure 2A and 2B) was taken of each eye, and at first glance it may appear to have 
the same inferior thinning and steepening as seen in the prior pre-operative topography. However, postoperatively the topography had altered significantly. His right keratometry readings went from 53.3/48.8@ 058 to 64.1/59.4@157 and left went from 54.0/43.1@ 103 to 56.6/56.3@179. The thinnest corneal reading went from 348um to $365 \mathrm{um}$ in the right eye and 452 um to 418 um in the left eye. Both sets of INTACS and CXL essentially flipped the cylinder correction. Hence, reduced the overall astigmatism dramatically by $10.6 \mathrm{D}$ in the left eye, however the right eye increased slightly by $0.2 \mathrm{D}$. Interestingly, the thinnest corneal measurement increased by $17 \mathrm{um}$ in the right eye but decreased by $34 \mathrm{um}$ in the left eye after CXL. Also, the overall anterior chamber volume measurements decreased from $239 \mathrm{~mm}^{3}$ to $218 \mathrm{~mm}^{3}$ in the right eye and $244 \mathrm{~mm}^{3}$ to $217 \mathrm{~mm}^{3}$ in the left eye. Finally, it is important to recognize the quality specification was flagged and may account for reading errors.

The differential diagnosis for this patient with a history of INTACS with CXL were post-surgical ectasias, keratoconus, PMD, keratoglobus, posterior keratoconus and contact lens induced corneal warpage. At first glance and evaluation, the current topography suggests that this is a severe form of typical inferior steepened and thinned corneas as seen with keratoconus with superior flattening. No Fleischer ring of corneal epithelial iron deposits at the base of the cones were observed. A positive Munson's sign was demonstrated by the protrusion of his lower eyelids on downward gaze. Upon further investigation, a topography (Figure 3) was discovered on August $14^{\text {th }}, 2007$ demonstrating signature pellucid marginal degeneration (PMD) or peripheral corneal degeneration OU.

FIG. 2A Post-operative OD topography.
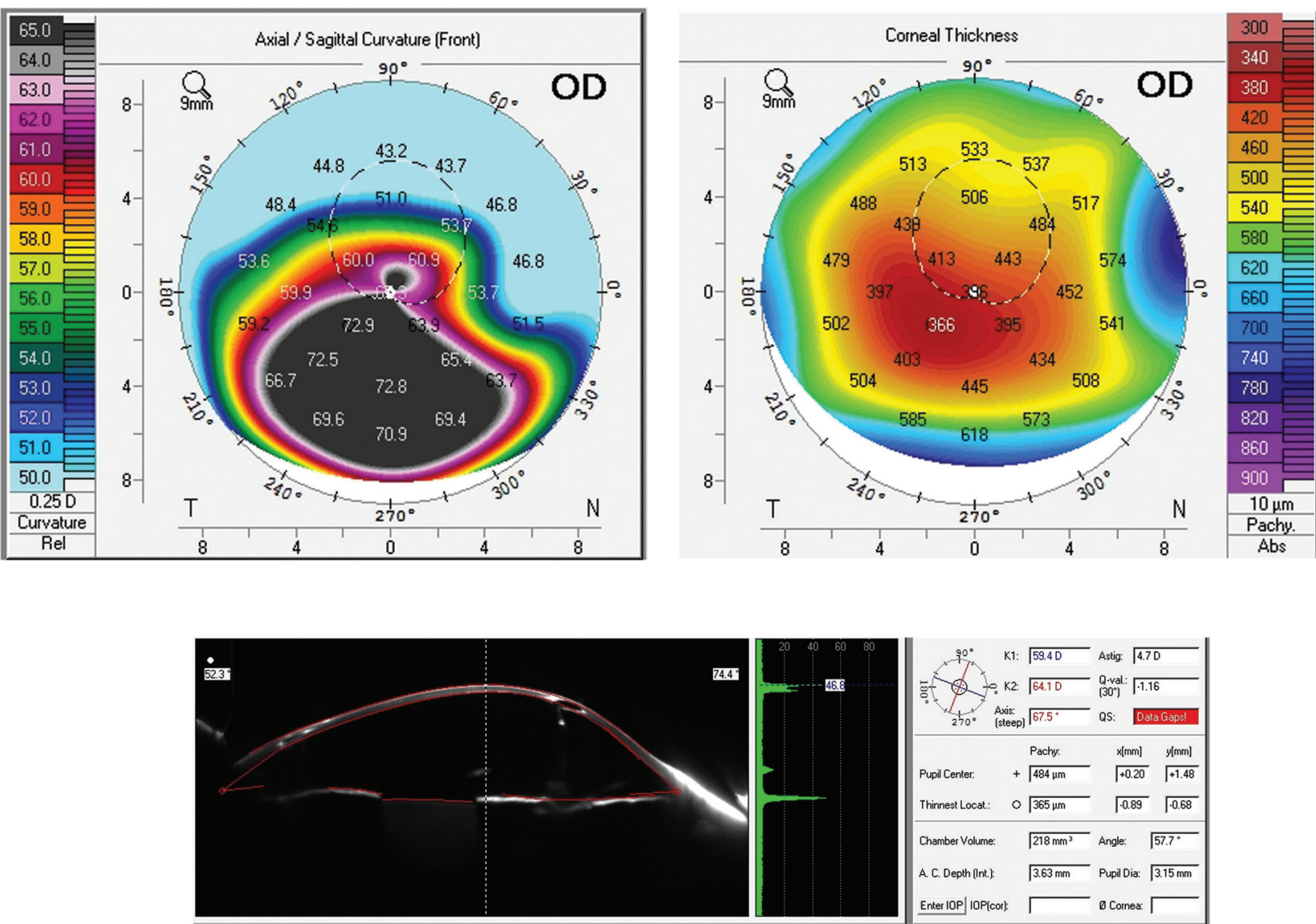

J Cont Lens Res Sci Vol 2(1):1-14; January 19, 2018. @ 2018 Journal of Contact Lens Science and Research. All rights reserved. This article is distributed under the terms of the Creative Commons Attribution-Non Commercial 4.0 International License. 
FIG. 2B Post-operative OS topography.
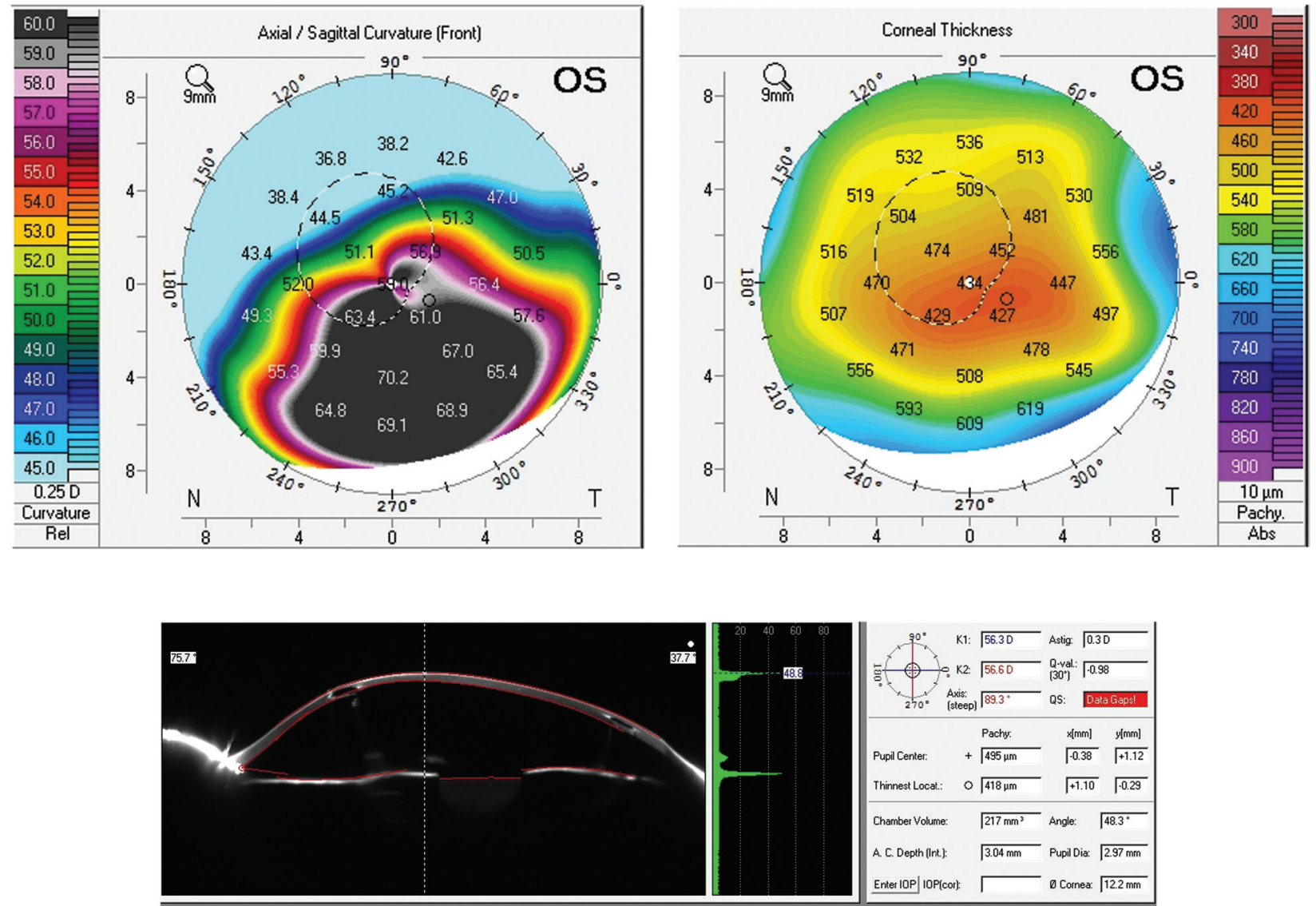

The patient's topography was observed to have patterns of "birds kissing," or a "crab claw" with significant invagination along the flat axis of the cornea, oblate in shape, and the peak elevation index (PEI) is over $3 \mathrm{~mm}$ from the corneal apex OU. ${ }^{7}$ The PEI is determined by measuring the highest elevation to the corneal apex. The average PEI for $\mathrm{KC}$ is about $1.95 \mathrm{~mm}$ while the PEI for PMD is an average of $3.5 \mathrm{~mm} .^{7} \mathrm{He}$ did not demonstrate any signs of postsurgical corneal ectasia, perforation, or inflammation. Although the patient did exhibit inferior corneal thinning and steepening, he did not exhibit the rare, globular, blistered shape of keratoglobus. The patient was not diagnosed Ehlers-Danlos syndrome that is associated with keratoglobus. Posterior keratoconus demonstrating a posterior corneal island pattern was not observed with this patient. Lastly, the patient has been wearing well-fitted scleral contact lenses on and off for the past several months which rules out contact lens induced corneal warpage.

The patient was diagnosed with advanced PMD with successful INTACS and CXL due to the crabclaw corneal topographies, significant inferior stromal thinning with striae and small mild round scarring slightly inferior of both corneas. Although $\mathrm{KC}$ and PMD are thought to be 2 separate diagnoses, there has been no evidence supporting these are in fact different ectatic processes.

Medically indicated scleral lenses were recommended for the patient to improve visual acuity, comfort, and therapeutically reduce corneal dryness. Spectacles were an inferior option. Scleral lenses were warranted due to the severe state of his disease and to complete the treatment recommended by the 
FIG. 3 Discovery of signature pellucid marginal degeneration (PMD) or peripheral corneal degeneration OU.

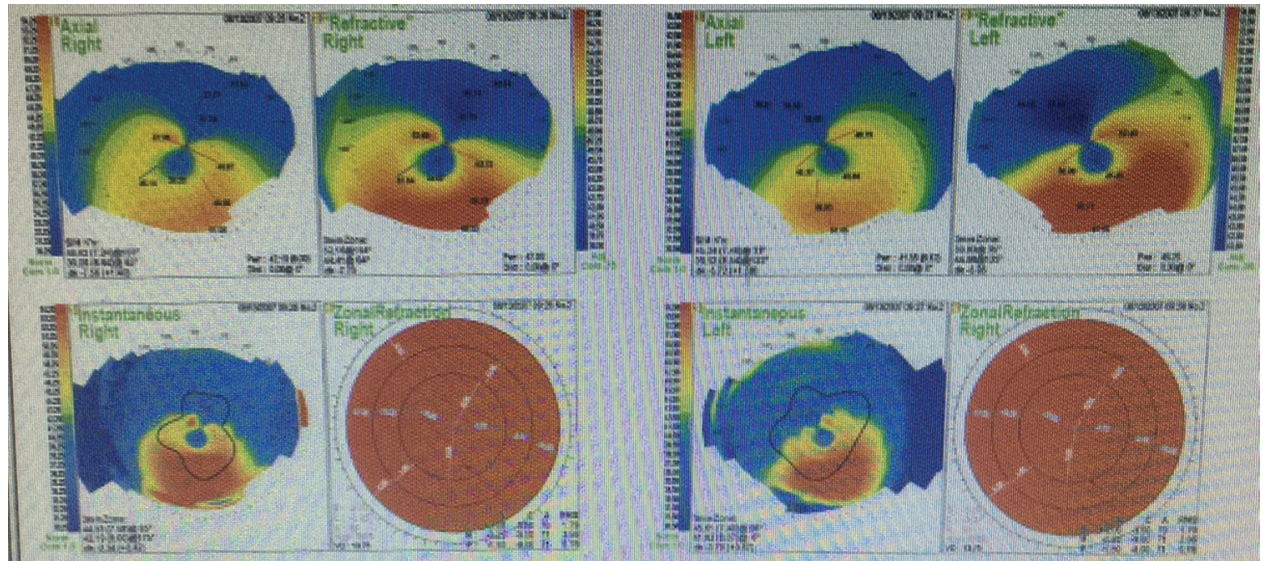

corneal specialists. The specialists and the patient's plan was to obtain the best fitting scleral lenses to reduce the likelihood of corneal transplants. Due to the progression observed, the plan was to stabilize his corneas with INTACS and CXL and then prescribe contacts to provide the best visual acuity and long-term therapy. Since the patient was still motivated and had a considerably positive experience with scleral lenses, the Zenlens was recommended. Although custom soft lenses, piggyback systems, or hybrid lenses would also be an option, the patient preferred to continue with scleral GP lenses.

Following the Zenlens manufacture guidelines, the Z-21 were the initial lenses chosen. Due to the patient's measured HVID being greater than $11.8 \mathrm{~mm}$ OU, the larger, $17 \mathrm{~mm}$ diagnostic lens was used. Since the patient's corneas were steeper in the periphery, the oblate lens design was elected. The following lenses were filled with preservative free saline solution and inserted into the patient's eyes using insertion plungers without complications or bubbles:

OD: Z-21: BC: $9.70 \mathrm{~mm}$, Dia: $17.0 \mathrm{~mm}$, Pwr: -2.00, SAG: 4800 um, PC: Standard

OS: Z-21: BC: $9.70 \mathrm{~mm}$, Dia: $17.0 \mathrm{~mm}$, Pwr: -2.00 , SAG: 4800 um, PC: Standard

The lenses were assessed after approximately 5 minutes. Both of the lenses demonstrated inferior mid-peripheral bearing with the right lens appearing to have slightly less clearance. Inadequate sagittal depth of the lenses directed the second trial lenses to be steeper. The following trial lenses were inserted:

OD: Z-23: BC: $8.50 \mathrm{~mm}$, Dia: $17.0 \mathrm{~mm}$, Pwr: -2.00, SAG: 5400 um, PC: Standard

OS: Z-24: BC: $7.90 \mathrm{~mm}$, Dia: $17.0 \mathrm{~mm}$, Pwr: -2.00, SAG: 5700 um, PC: Standard

The lenses were evaluated and exhibited slight inferior centration with improved central and midperipheral clearance. The estimated clearance over the steepest corneal elevation was $160 \mathrm{um}$ for the OD and 264 um for the OS. Both eyes minimally cleared the limbus without touch and displayed tight horizontal edges with mild compression and blanching of the nasal aspect of the conjunctiva. Over-refraction of the right eye was $-2.75-1.00 \times 150$ to $20 / 20$ and $-5.25 \mathrm{DS}$ to $20 / 20$. About 15 minutes post insertion, anterior segment ocular coherence tomography (ASOCT) was collected as seen below in Figures 4A and 4B.

The patient was impressed with the quality of the vision and increase in overall comfort in comparison to the PROSE lens. After allowing the lens to settle for over 45 minutes, the lenses were assessed again. Due to minimal sagittal depth on the right eye, the vault was increased by 300 um that made the total SAG: 5700 um. The Zenlens Smart Curve allows independent changes to the sagittal depth without changing the base curve. The minimal horizontal limbal clearance and the slight inferior decentration was addressed by adding toric peripheral curves and 
FIG. 4A Right ASOCT.
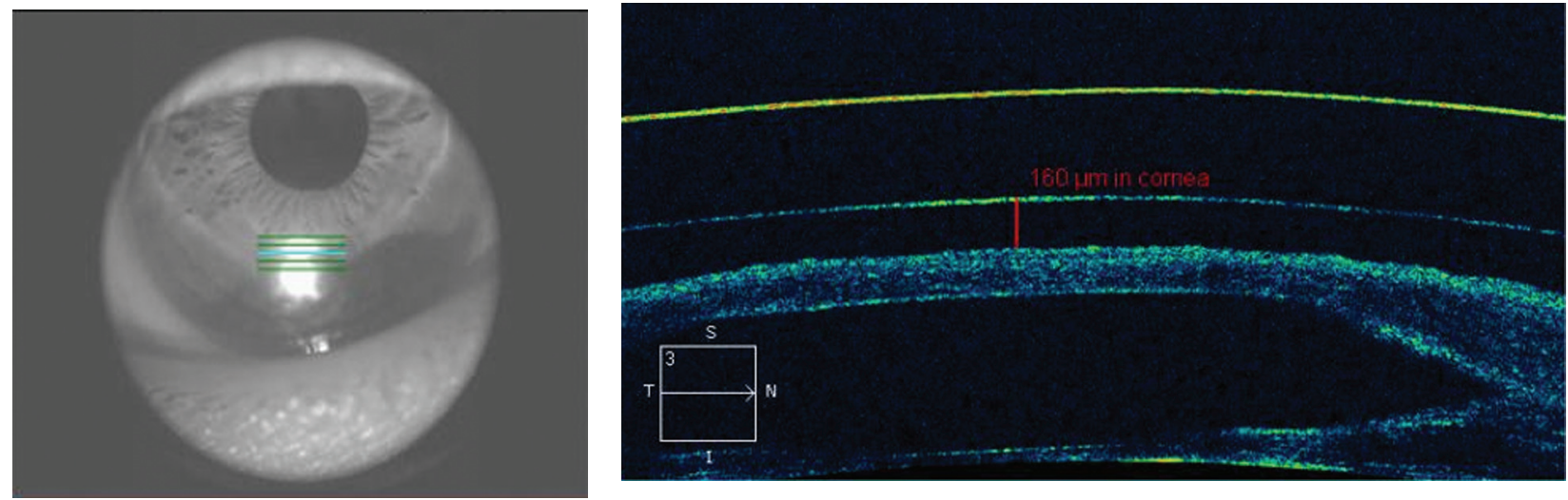

FIG. 4B Left ASOCT.
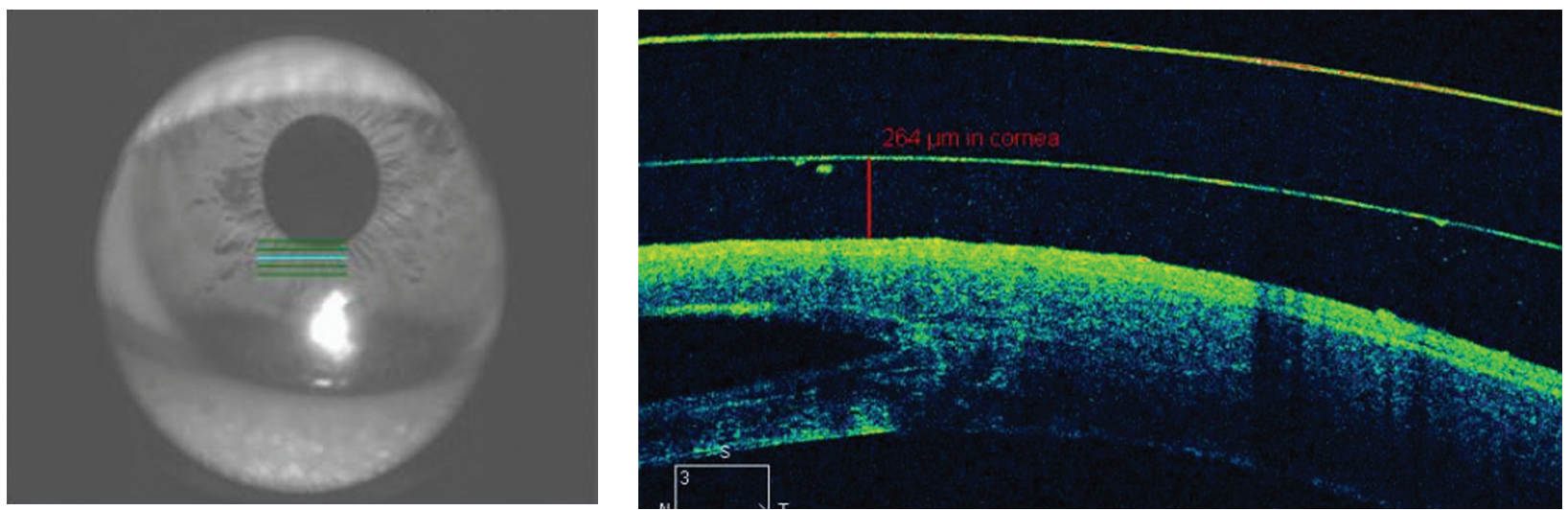

increasing the limbal curve by $150 \mathrm{um}$ in each eye. The power of the lenses was calculated using the overrefraction. The goal was to achieve complete vault over the entire cornea with a target of 250-350 um over the steepest corneal elevation, adequate limbal clearance 360 degrees, good centration, minimal to no movement, adequate landing, and optimal vision correction. The following final lenses were ordered in attempt to achieve the goal stated above:

OD: Zenlens, BC: $8.50 \mathrm{~mm}$, Dia: $17.0 \mathrm{~mm}$, Pwr: $-4.75-1.00 \times 150$, SAG: 5700 um, LZ: +150 um PC: flat 3 horizontal / steep 1 vertical, Boston XO mate-rial, single dot @ 6 o'clock, blue tint

OS: Zenlens, BC: $7.90 \mathrm{~mm}$, Dia: $17.0 \mathrm{~mm}$, Pwr: -7.00, SAG: 5700 um, LZ: +150um, PC: flat 4 horizontal / steep 1 vertical, Boston XO material, double dot OS@6o'clock, blue tint

The patient was scheduled to return in one month for a contact lens evaluation with expectations to dispense. Since the patient demonstrated good technique and had experience with sclerals the patient did not need additional training.

\section{DISPENSING VISIT}

The patient presented on November $29^{\text {th }}, 2016$ for his dispensing visit. The new lenses were filled with preservative free saline solution and inserted on the eyes using insertion plungers without complications or bubbles and were assessed after approximately 5 minutes. The patient reported acceptable lens comfort

J Cont Lens Res Sci Vol 2(1):1-14; January 19, 2018. @ 2018 Journal of Contact Lens Science and Research. All rights reserved. This article is distributed under the terms of the Creative Commons Attribution-Non Commercial 4.0 International License. 
and vision. The visual acuity was measured to be 20/20 in the right and left eye and 20/15 - with both eyes open. An over-refraction was completed and was found to be PL $-0.50 \times 005$ in the right eye and PL $-0.50 \times 020$ in the left eye providing no significant visual improvement.

Slit lamp evaluation revealed both lenses to have adequate centration with an estimated central clearance of approximately $356 \mathrm{um}$ in the right eye and $248 \mathrm{um}$ (Figure 5A and 5B) in the left eye. Both lenses cleared the limbus (Figure 6A and $6 \mathrm{~B}$ ) and provided acceptable edge alignment without any signs of compression or impingement (Figure 7). When manually rotated, the lenses returned back to the original position with the dots at the desired position due to the toricity of the lenses. Both lenses were found to be acceptable for dispensing as the fit, feel and vision were appropriate. Both eyes fluorescein stained $1+$ scattered punctate epithelial keratopathy. No other ocular findings were remarkable.

Patient education concerning the wear and care was provided. Peroxide-based cleaning solutions and non-preserved saline solution for lens insertion was recommended. The patient was provided a lens care kit that included insertion and removal plungers, a mirror with stand, a pen light to look for bubbles after insertion, and a large scleral lens case for over-night cleaning using Clear Care ${ }^{\circledR}$. Proper insertion and removal education was reinforced and was instructed to slowly increase the wear time of the lenses over the next several weeks, not to exceed twelve hours until the scheduled follow-up in 3 weeks before the holiday.

\section{FOLLOW-UP \# 1}

The patient presented in the clinic on December $22^{\text {nd }}, 2016$ for his contact lens progress evaluation. He

FIG. 5A Right ASOCT.
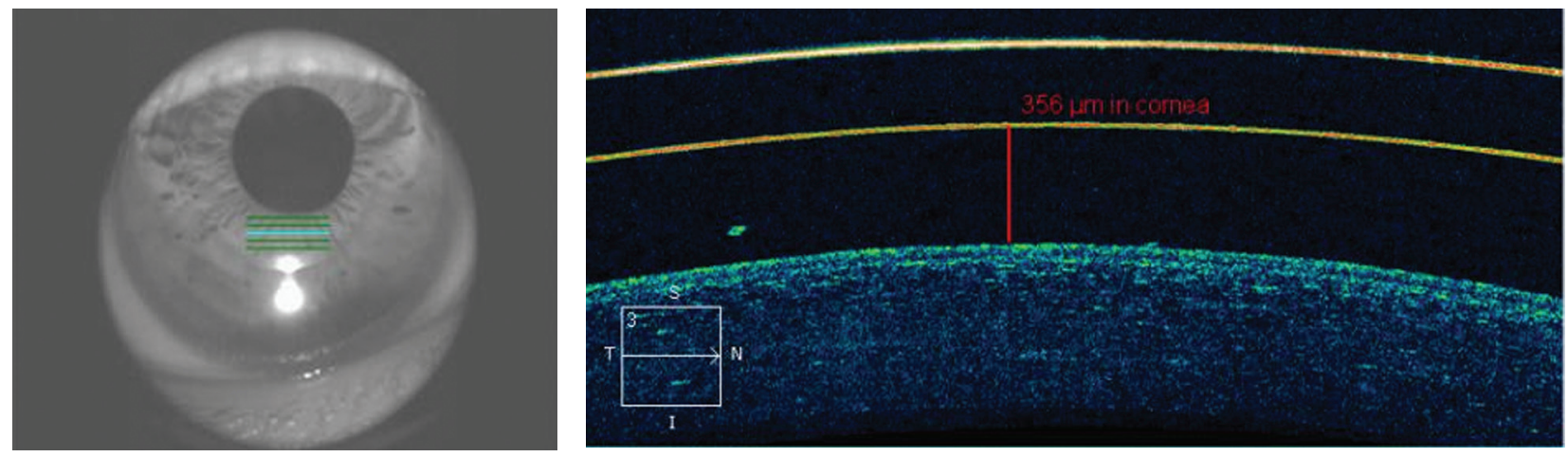

FIG. 5B Left ASOCT.
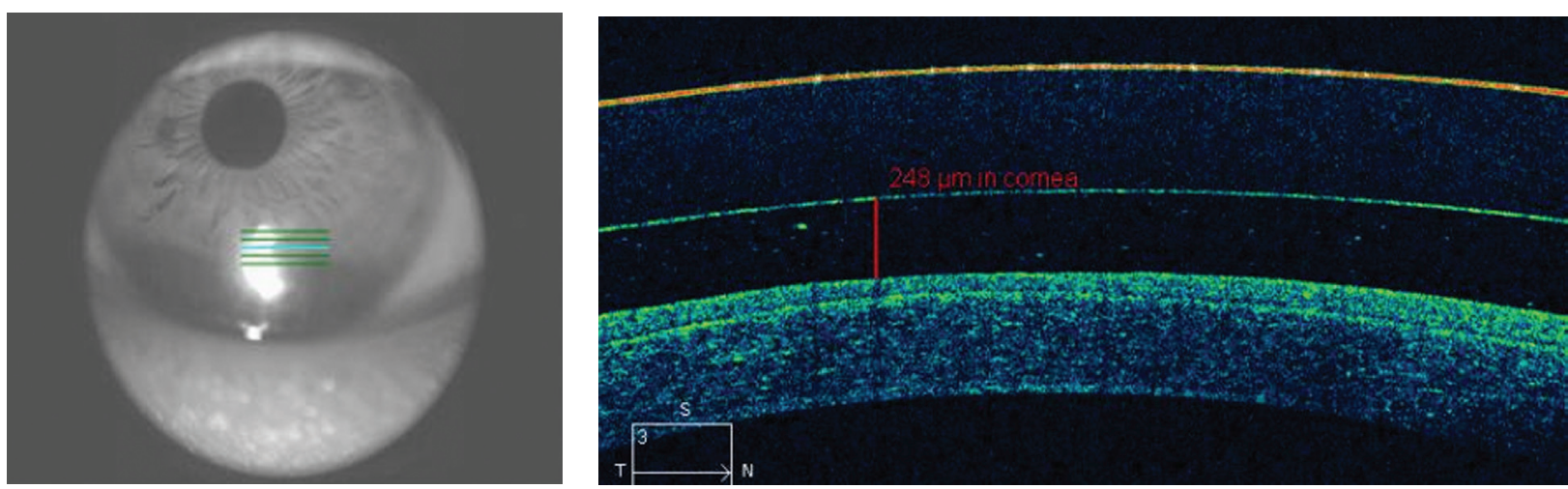

J Cont Lens Res Sci Vol 2(1):1-14; January 19, 2018. @ 2018 Journal of Contact Lens Science and Research. All rights reserved. This article is distributed under the terms of the Creative Commons Attribution-Non Commercial 4.0 International License. 
FIG. 6A Limbal Comparison Oct. $14^{\text {th }}$, 2016 OS Temporal ASOCT.
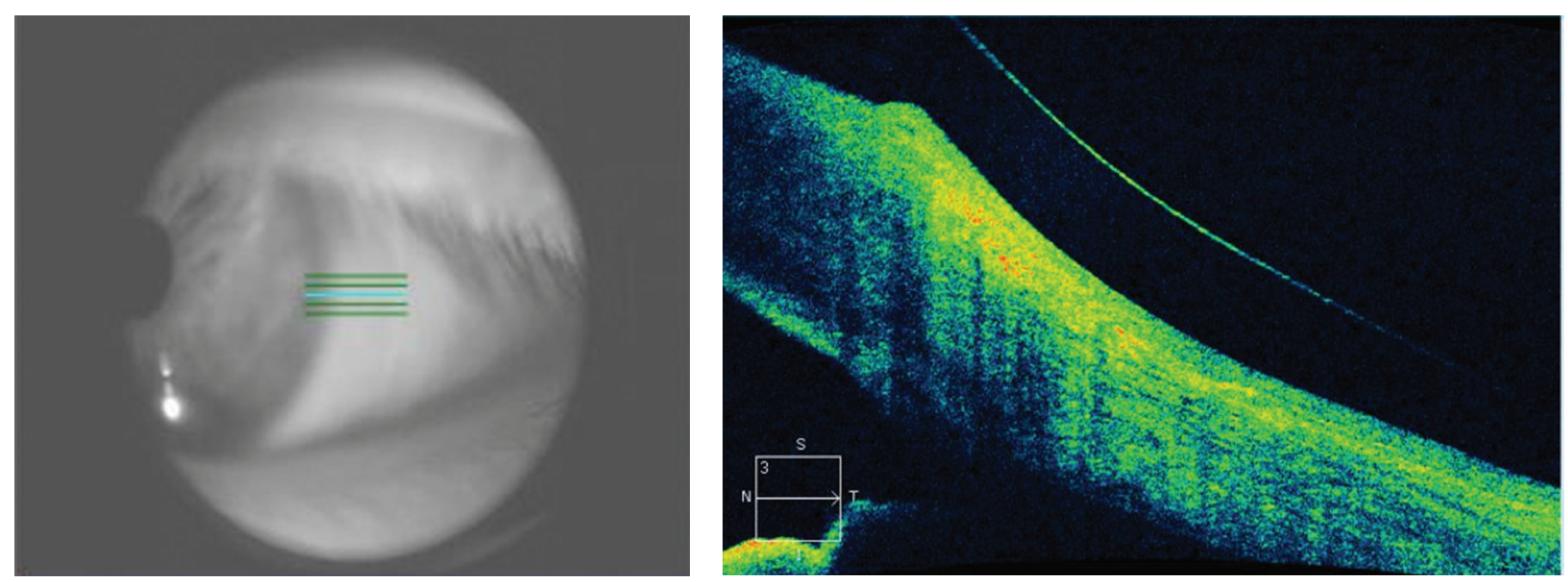

FIG. 6B Limbal Comparison Nov. 29 ${ }^{\text {th }}, 2016$ OS Temporal ASOCT.
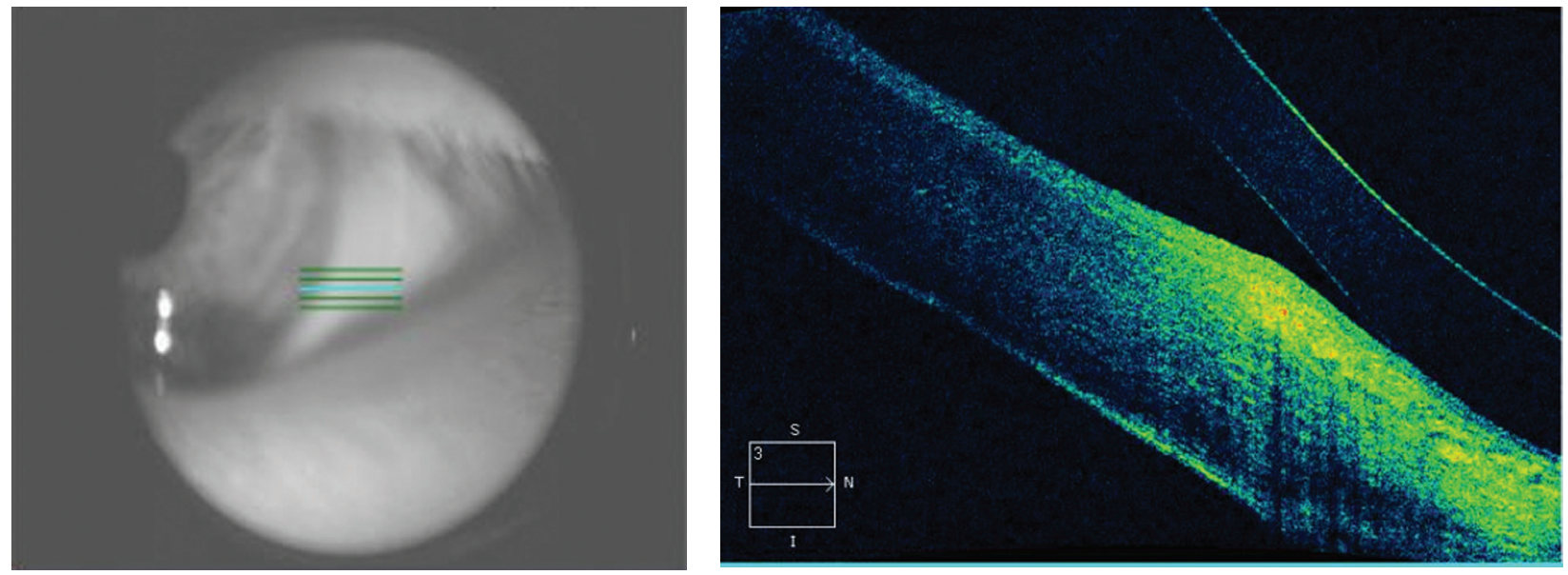

reported great vision and comfort was great in both eyes with no issues managing the lenses. He specifically expressed that he liked these lenses the best due to the vision, comfort, and was able to use them full time for at least twelve hours per day.

The visual acuity was measured to be $20 / 20+$ in the right and left eye and 20/15 with both eyes open. There was no significant over-refraction. Upon slit lamp evaluation, both lenses revealed adequate centration. Both lenses showed an estimated central clearance over 240 um and cleared the limbus. The lenses provided acceptable limbal clearance and edge alignment without any signs of compression or impingement.
The patient's anterior segment revealed no punctate epithelial keratopathy or any other remarkable ocular findings were observed. The patient was instructed that he may continue wearing the lenses during the day but never to sleep or swim in them. It was requested the patient be scheduled for a one-month follow-up to assess lenses.

\section{FOLLOW-UP \#2}

The patient presented in the clinic on March $21^{\text {st }}$, 2017 for a contact lens progress evaluation. He reported his vision and comfort was great in both eyes with no issues managing the lenses. He has been using them

J Cont Lens Res Sci Vol 2(1):1-14; January 19, 2018. @ 2018 Journal of Contact Lens Science and Research. All rights reserved. This article is distributed under the terms of the Creative Commons Attribution-Non Commercial 4.0 International License. 
FIG. 7 Left image: OD. Right image: OS.

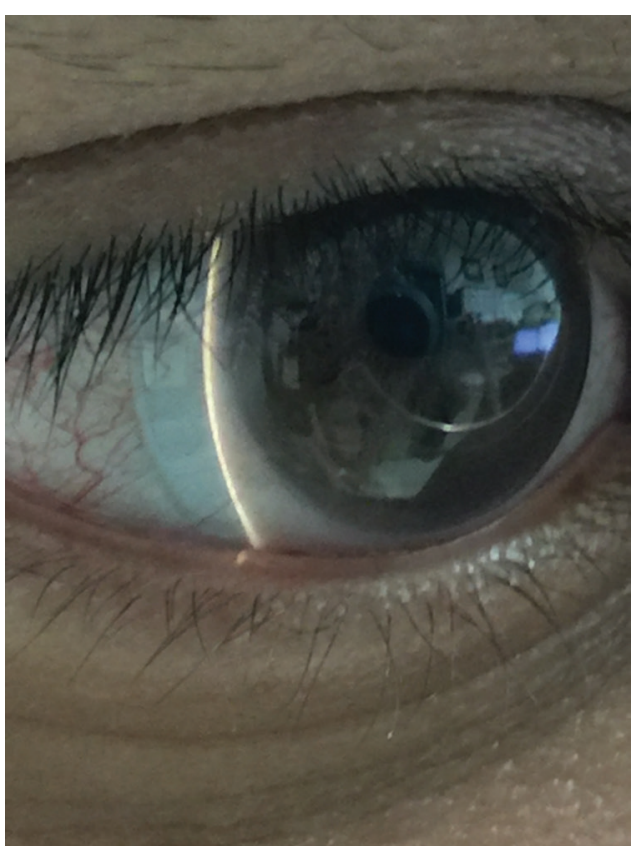

full time for at least twelve hours per day. The visual acuity was measured to be 20/20 in the right and left eye and 20/15 with both eyes open.

The lenses were filled with preservative free saline solution and inserted into the patient's eyes using insertion plungers without complications or bubbles and were assessed after approximately 5 minutes. The visual acuity was measured to be $20 / 20$ in the right and left eye and 20/15 - with both eyes open. An over-refraction was of plano was obtained in each eye providing $20 / 20+$ to each.

Upon slit lamp evaluation, both lenses revealed excellent centration. Both lenses showed an estimated central clearance over 240 um and cleared the limbus. Both lenses provided acceptable edge alignment without any signs of compression or impingement. Although it is not clinically significant, it is important to recognize the hyperemia outside the temporal edges of the lenses (see Figure 7). No punctate epithelial keratopathy or new ocular findings were observed compared to the last exam. The patient was instructed that he may continue to wear the lenses full time during the day but never to sleep or swim in them. The patient was scheduled for a one month follow-up.

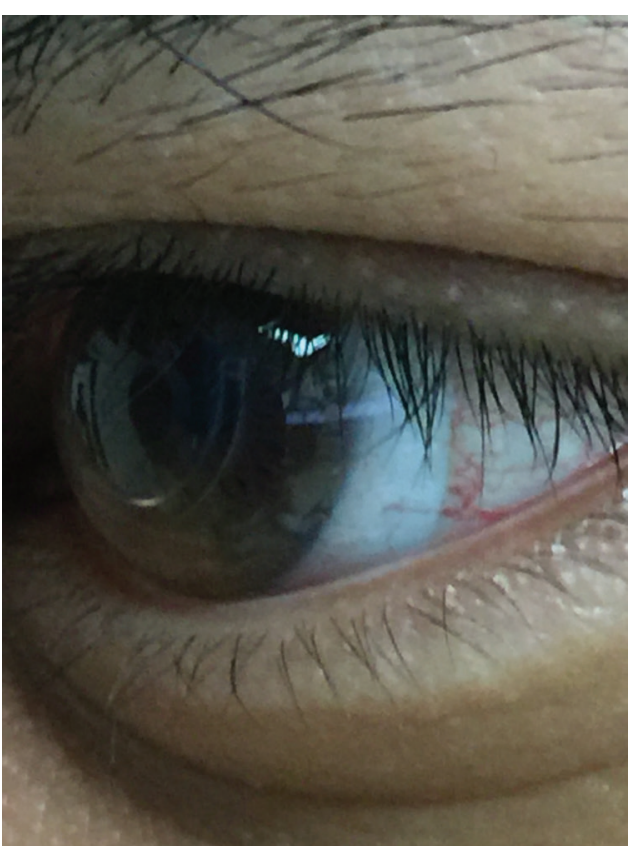

\section{FOLLOW-UP \#3}

The patient presented in the clinic on April $12^{\text {th }}$, 2017 for contact lens evaluation. He reported his vision and comfort was great in both eyes with no issues managing the lenses. He added that he has been using them full time for at least twelve hours per day. The visual acuity was 20/20+ in the right and left eye and 20/15 with both eyes open. An over-refraction was completed and was found to be plano in each eye providing $20 / 20+$ to each.

Upon slit lamp evaluation, both lenses revealed excellent centration. Both lenses showed an estimated central clearance over $240 \mathrm{um}$ and appeared to clear the limbus (Figure 8A and 8B).

Complete corneal and limbal clearance was observed. Both lenses provided acceptable edge alignment without any signs of compression or impingement. Anterior segment revealed 1+ conjunctival injection of both eyes, significant inferior stromal thinning with striae and small mild round scaring slightly inferior of both corneas. The INTACS in both eyes appeared well placed and without signs of complication. Temporal limbal neovascularization measured to be $1 \mathrm{~mm}$ of 
FIG. 8A OD Anterior Segment Pentacam.
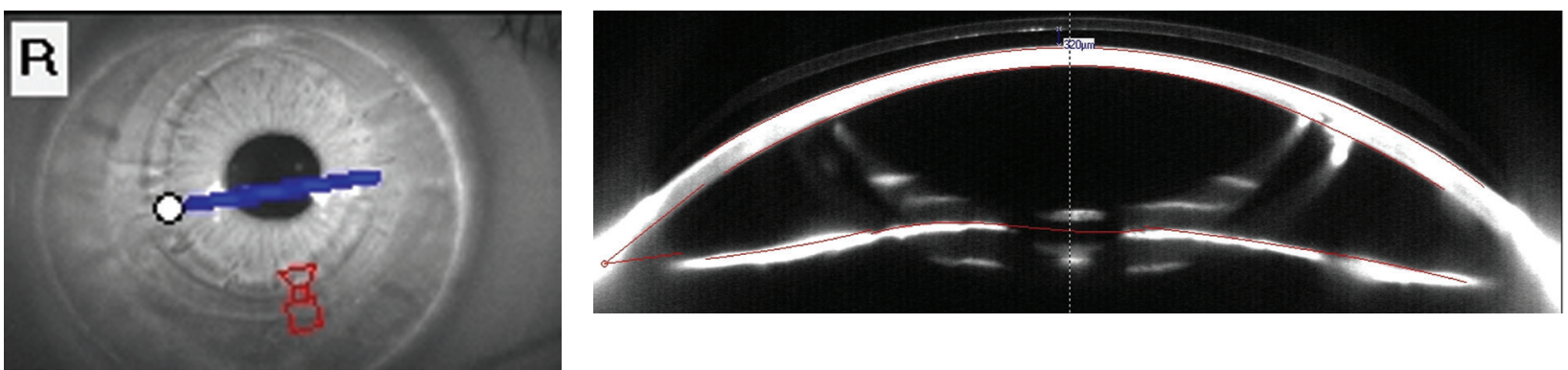

FIG. 8B OS Anterior Segment Pentacam.
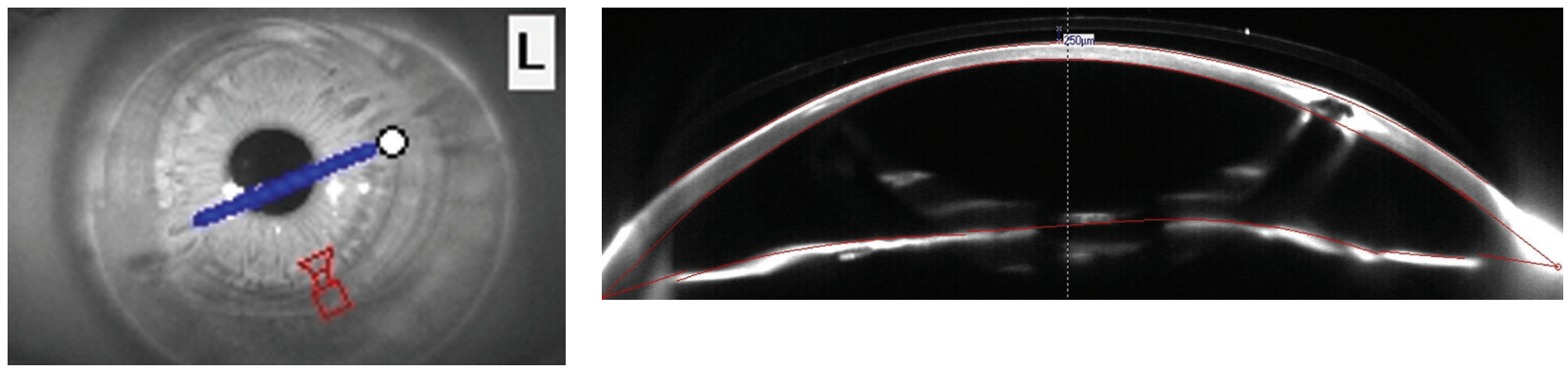

encroachment of the left eye only were observed. No punctate epithelial keratopathy or any other remarkable ocular findings were observed. The patient was instructed to continue utilizing the lenses and wear them full time during the day but never to sleep or swim in them.

No alterations were necessary to the lenses since there were no clinically significant new findings or changes since the last exam. His contact lens prescription was finalized and was instructed to follow-up in 6 months for a comprehensive eye exam and evaluation of the sclerals.

\section{DISCUSSION}

PMD is a rare, progressive, and degenerative corneal disease typically characterized by peripheral, bilateral, and inferior ectasia producing a crescent shape. ${ }^{1} \mathrm{PMD}$, while rare, is the second most common non-inflammatory corneal thinning disorder behind keratoconus. ${ }^{1,8,9}$ It has been found more commonly in males and appears between the second and fifth decade of life showing no signs of ethnicity predilection. ${ }^{1,8}$ It is possible PMD is more common than reported because of the lack of practitioners reporting the difference. PMD is commonly diagnosed as $\mathrm{KC}$ or a version of $\mathrm{KC}$, therefore not well differentiated. ${ }^{1,9}$ Although these conditions are clinically different, they may be from the same ectatic process that is not yet fully understood.

$\mathrm{KC}$ is a developmental anomaly in which a part of the cornea becomes thinner and bulges forward in a cone-shaped fashion as a result of non-inflammatory stromal thinning. ${ }^{2} \mathrm{KC}$ is predominantly bilateral $(90 \%)$ and according to recent studies demonstrates a prevalence of $2.3 \%{ }^{2,3}$ The CLEK study found the genetic predisposition of $\mathrm{KC}$ to be at $13.5 \%$ whereas other studies found it to be between $6-10 \%{ }^{2,3}$ This indicates most of those found having $\mathrm{KC}$ have no known association with the disease and that we have not yet truly determined the cause. Regardless, KC and PMD are complex diseases where eye rubbing has been associated with $\mathrm{KC}$ and is considered an additive environmental factor.

Pathophysiology of PMD has not been discovered. It could be due to collagen abnormalities causing stromal thinning and an irregular Bowman's membrane..$^{5,8,9}$ 
Corneal stromal thinning is apparent in the topographies of the patient (see Figures 1 and 2). One case study showed that PMD was coexistent with corneal plana in an individual with a "KERA" mutation, thus, potentially implicating keratocan as a possible factor in the development of PMD. ${ }^{10}$ Keratocan is a protein found uniquely in the cornea linked to assisting in corneal transparency. Many studies have been done to examine the biomechanic and genetic expression of this disease. Although it remains unclear, some studies are suggesting genetic loci and variants have been implicated in the genetic susceptibility of this disease. ${ }^{5,9,10}$

Once identified, PMD and $\mathrm{KC}$ have similar treatment options. It is typical for less severe cases of PMD to be treated with spectacles and toric soft contact lenses. More severe PMD patients may require more specialized options such as custom soft contacts, rigid GP lenses, or hybrid contact lenses. Depending on the severity of the corneal thinning, the clarity of the cornea and the amount of irregular astigmatism, the visual potential and quality of vision can range widely. Monocular diplopia, ghosting, blurring, and image distortion are all complications of astigmatism that may arise from PMD. These complications may all be mitigated through specialty scleral GPs that offer excellent oxygen permeability to maintain the health of the cornea. Corneal GPs are not typically used for PMD due to the inferior peripheral steepening that the edges of the GP may land on. This may cause excessive movement, inhibit comfort, and increase the potential of corneal scarring.

Scleral GPs that are fit appropriately provide 360 degrees of corneal and limbal coverage and a vault that rests on the conjunctiva and sclera. ${ }^{12}$ Due to the size of the lens and the location of the landing zone, the lens awareness or irritation is minimal. As seen with patient \#2, the sclerals provided optimal vision correction that could not be obtained with spectacles. The lenses provided excellent dry eye therapy and resolved the punctate epithelial keratopathy completely. The patient had no discomfort and was able to wear the lenses all day.

Poor fitting scleral GPs may cause complications. Microbial keratitis, corneal keratitis and corneal scarring are among the rare complications reported. ${ }^{12}$
Lens fogging, or mid-day haze is a more common complication that is associated with ocular surface disease, a poorly fitting lens, or debris within the tear lens. ${ }^{12}$ The ocular surface disease may be improved by continuous daily wear of the sclerals, as seen by patient \#2. Otherwise, improving the fit by aligning the scleral landing and ensuring no bubbles or debris on insertion can prevent fogging. Extremely poor fitting scleral GPs may cause corneal edema and neovascularization. However, it has been reported that neovascularization may improve with the use of properly fitting sclerals, which was the intent for the patient. ${ }^{12}$

In severe PMD or KC such as this case, surgical treatments may be warranted. In the most severe cases, penetrating keratoplasties (PKP) have shown great success. Although, they typically require spectacle or contact lens correction afterwards and there is a clinically significant risk of graft rejection. ${ }^{13,14}$ According to the Corneal Donor Study, the graft age was not found to be a rejection factor. The 10-year graft failure rate was $21 \%$ from all causes, $37 \%$ in cases with pseudophakic or aphakic corneal edema and 20\% in cases with Fuchs' dystrophy. Furthermore, in cases with prior glaucoma surgery, a graft failure rate of $58 \%$ was found versus $22 \%$ with no history of glaucoma. ${ }^{14}$

The FDA approval of $\mathrm{KC}$ treatment using corneal crosslinking (CXL) in April 2016 has shown excellent promise of improving the visual potential of patients with this progressive disease. ${ }^{4,13} \mathrm{CXL}$ with riboflavin and ultraviolet $\mathrm{A}$ is a technique used to strengthen corneal tissue with riboflavin as a photosensitizer and ultraviolet $A$ to increase the formation of stromal collagen cross linking by photosensitized oxidation. ${ }^{15}$ Riboflavin (vitamin $\mathrm{B}_{2}$ ) has a dual function of acting as a photosensitizer for the production of oxygen free radicals, which induce physical cross linking of collagen, and it gives a protective effect by absorbing $90 \%$ of the UV-A $(370 \mathrm{~nm})$ irradiation thereby preventing damage to deeper ocular structures. ${ }^{16}$

The FDA has approved this procedure that typically begins with corneal epithelial debridement. The Dresden Protocol has been named a standard corneal cross-linking procedure. ${ }^{4}$ The protocol includes the installation of $0.1 \%$ riboflavin solution in

J Cont Lens Res Sci Vol 2(1):1-14; January 19, 2018. @ 2018 Journal of Contact Lens Science and Research. All rights reserved. This article is distributed under the terms of the Creative Commons Attribution-Non Commercial 4.0 International License. 
$20 \%$ dextran for 30 minutes with drops every $3-5$ minutes, followed by UV-A irradiation with $3 \mathrm{~mW} /$ $\mathrm{cm}^{2}$ for 30 minutes for a total dose of $5.4 \mathrm{~J} / \mathrm{cm}^{2}$. A bandage soft contact lens is then utilized to aid healing and minimize pain in conjunction with a topical antibiotic and anti-inflammatory post-surgical regiment. There are numerous protocols using different riboflavin formulations (trans-epithelial, nondextran, and different concentrations of riboflavin) and different levels of UV-A illumination being studied. ${ }^{4,15,16} \mathrm{CXL}$ is being assessed to be used for other conditions, such as post-LASIK regression minimization, treatment of infections keratitis, corneal melts, Acanthamoeba cysts, and post-thermal keratoplasty stabilization. ${ }^{4,15,16}$

Some CXL studies reported associated complications and limitations. For example, it is not recommended in cases with a corneal thickness of $400 \mathrm{~nm}$ or less because of increased risk of corneal endothelial cell damage. ${ }^{4,20}$ Also, postoperative microbial keratitis, corneal haze, corneal endothelial damage, sterile corneal stromal damage, herpes simplex virus reactivation, and treatment failure/keratoconic progression are among the reported of complications of CXL. ${ }^{4,20}$ It has been shown that CXL is able to reduce corneal steepening, improve refractive error by minimizing irregular astigmatism, can inhibit the progression of corneal ectasia with a low risk of complications, and reduce the need for keratoplasties. ${ }^{4,13,15,16,20}$

Another surgical option approved by the FDA in July 2004 that the patient took advantage of is called intra-stromal corneal ring segments or INTACS. Clear crescent plastic rings are inserted into the stroma on a specific steep axis to flatten the overall corneal curvature. ${ }^{17}$ As seen with patient $\# 2$, this was an additive surgery due to his severe amount of astigmatism. Clearly demonstrated in Figures 1 and 2, the procedures essentially flipped the cylinder correction, reduced the overall astigmatism dramatically by $10.6 \mathrm{D}$ in the left eye and the right eye increased slightly by $0.2 \mathrm{D}$.

There are many versions of protocols used to apply INTACS. Due to complications observed over time, newer procedures have been applied. For example, the use of IntraLase laser created channels have reduced the complications of false channels, perforations, and may provide very precise and accurate channels as compared to a manual approach. ${ }^{17,21}$ Another protocol suggested 2 equal ring segments should be used for symmetric bow-tie patterns of ectasia or keratoconus and asymmetrical segments should be used for peripheral cones and the segment thickness should not exceed $50 \%$ of the thickness of the cornea. ${ }^{21}$ Postoperative microbial keratitis, peripheral corneal haze, peripheral corneal endothelial damage, sterile corneal stromal damage, and treatment failure/keratoconic progression are among the reported of complications of INTACS. ${ }^{17,21,22}$

One study documented long-term ( $>5$ years) results of KeraRings intracorneal ring implantation and concluded that KeraRings result in topographic and visual stability, delayed the progression of keratoconus and at the very least postponed the need for keratoplasty. ${ }^{21}$ Of note, regression of visual stability and progression of keratoconus has been observed a year or longer after INTACS. It was concluded that ectatic corneas with high irregularity and a pronounced conic protrusion may be poor candidates for intracorneal ring segment implantation. ${ }^{21}$ Those with highly irregular and advanced ectasias may benefit from combined INTACS and CXL surgeries. ${ }^{21,22}$

Further evidence of this surgical combination is being evaluated and more long-term treatment strategies are recommended. ${ }^{22}$

Although the procedures were deemed successful by ophthalmology due to the lack of complications and reduction of corneal steepening, he required contact lenses to achieve the best vision. Continued evaluation over the next several years will determine stabilization. With the addition of scleral contacts, he and his eye care team is hopeful these treatments will stabilize his PMD and prevent further ectatic corneal progression and prevent him needing PKP procedures. Since PMD can cause severely abnormal corneal topography, the treatment can be very challenging and may require multiple specialists and treatments. One study found that $88 \%$ of PMD cases were managed non-surgically with spectacles $(36 \%)$ or contacts $(52 \%)$, whereas $12 \%$ underwent PKP. ${ }^{18}$

\section{CONCLUSION}

Dating back to 1610 , the term pellucid comes from a Latin word pellūcidus, which translates to shine through or transparent. ${ }^{19}$ The condition was named

J Cont Lens Res Sci Vol 2(1):1-14; January 19, 2018. @ 2018 Journal of Contact Lens Science and Research. All rights reserved. This article is distributed under the terms of the Creative Commons Attribution-Non Commercial 4.0 International License. 
this because visual acuity can be healthy and the cornea typically appears transparent as the patients are usually asymptomatic except for progressive visual detertioration. ${ }^{18}$ Sometimes it's hard to imagine the optometric profession before the availability of corneal topography and OCTs. Although there is no cure for PMD or any of the versions of $\mathrm{KC}$, it is our job and responsibility as primary care optometrists to do our best to manage the condition and associated refractive complications. Proper patient education, identification and treatment are critical to the prognosis and treatment outcome of PMD. It is paramount to attempt to stabilize the condition and reduce the risk factors for loss of vision. This case demonstrates the usefulness of utilizing scleral GPs over INTACS post CXL to provide optimal vision, comfort and therapy.

\section{REFERENCES}

1. Jinabhai, Radhakrishnan H, and O'Donnell C. Pellucid corneal marginal degeneration: A review. Contact Lens Anter Eye 2011;34.2: 56-63.

2. Gordon-Shaag A, Millodot M, and Shneor E. The epidemiology and etiology of keratoconus. Epidemiology 2012;70:1.

3. Wagner H., et al. Collaborative Longitudinal Evaluation of Keratoconus (CLEK) Study: methods and findings to date. Cont Lens Anter Eye 2007;30(4):223-32.

4. Raiskup-Wolf F, et al. Collagen crosslinking with riboflavin and ultraviolet-A light in keratoconus: long-term results. J Cataract Refract Surg 2008;34(5):796-801.

5. Davidson AE, Hayes S, Hardcastle AJ, Tuft SJ. The pathogenesis of keratoconus. Eye 2014:28:189-95.

6. Schuerch K, Tappeiner C, and Frueh BE. Analysis of pseudoprogression after corneal cross-linking in children with progressive keratoconus. Acta Ophthalmol 2016;94(7).

7. Shovlin JP. Do I see keratoconus or PMD? How can we differentiate between these two similar ectatic diseases on corneal topography? Rev Optometry 2005;142(4):91-92.

8. Jinabhai A, Radhakrishnan H, O'Donnell C. Pellucid corneal marginal degeneration: A review. Contact Lens Anter Eye 2011;34(2):56-63.

9. Nagy M, Vigvary L: Etiology of the pellucid marginal degeneration of the cornea. Klin Monatsbl Augenheilkd 1972;161:604-611.
10. Khan AO, et al. Pellucid marginal degeneration coexistent with cornea plana in one member of a family exhibiting a novel KERA mutation. Br J Ophthalmol 2005;89(11):1538-40.

11. Barr JT, Wilson BS, Gordon MO, et al Estimation of the incidence and factors predictive of corneal scarring in the Collaborative Longitudinal Evaluation of Keratoconus (CLEK) Study. Cornea 2006;25:16-25.

12. van der Worp ECJ. A Guide to Scleral Lens Fitting, Version 2.0." Forest Grove, IL: 2015.

13. Schornack M, Patel S. Scleral lenses in the management of keratoconus. Eye Contact Lens 2010:1:39-44.

14. Sugar Alan. Factors associated with corneal graft survival in the cornea donor study." JAMA Ophthalmol 2015;133(3):246-54.

15. McCall AS, Kraft S, Edelhauser HF, et al. Mechanisms of corneal tissue cross-linking in response to treatment with topical riboflavin and long-wavelength ultraviolet radiation (UVA) Invest Ophthalmol Vis Sci 2010;51:129-38.

16. Arbelaez MC, et al. Collagen cross-linking with riboflavin and ultraviolet-A light in keratoconus: One-year results. Oman J Ophthalmol 2009;2(1):33.

17. Kanellopoulos AJ, et al. Modified intracorneal ring segment implantations (INTACS) for the management of moderate to advanced keratoconus: efficacy and complications. Cornea 2006;25(1):29-33.

18. Paulus, Y. Pellucid Marginal Corneal Degeneration. 2015; Available at: http://eyewiki.aao.org/ pellucid_marginal_corneal_degeneration

19. Harper D. Pellucidity. Online Etymology Dictionary. Available at: Dictionary.com http://www.dictionary. com/browse/pellucidity.

20. Hashemi $\mathrm{H}$, et al. Corneal collagen cross-linking with riboflavin and ultraviolet a irradiation for keratoconus: long-term results. Ophthalmology 2013;120(8):1515-20.

21. Coskunseven E, et al. Complications of intrastromal corneal ring segment implantation using a femtosecond laser for channel creation: a survey of 850 eyes with keratoconus. Acta Ophthalmol 2011:89(1):54-57.

22. Bromley JG and Randleman JB. Treatment strategies for corneal ectasia. Curr Opin Ophthalmol 2010;21(4):255.

J Cont Lens Res Sci Vol 2(1):1-14; January 19, 2018. @ 2018 Journal of Contact Lens Science and Research. All rights reserved. This article is distributed under the terms of the Creative Commons Attribution-Non Commercial 4.0 International License. 